\title{
Constructive techniques for zeros of monotone mappings in certain Banach spaces
}

\author{
C Diop $^{1}$, TM M Sow $^{1}$, N Djitte $^{1,2^{*}}$ and C E Chidume ${ }^{2}$
}

${ }^{*}$ Correspondence: ngalla.djitte@ugb.edu.sn

${ }^{1}$ Gaston Berger University, Saint Louis, Senegal

Full list of author information is available at the end of the article

\begin{abstract}
Let $E$ be a 2-uniformly convex real Banach space with uniformly Gâteaux differentiable norm, and $E^{*}$ its dual space. Let $A: E \rightarrow E^{*}$ be a bounded strongly monotone mapping

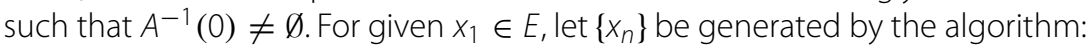

$$
x_{n+1}=J^{-1}\left(J x_{n}-\alpha_{n} A x_{n}\right), n \geq 1,
$$

where $J$ is the normalized duality mapping from $E$ into $E^{*}$ and $\left\{\alpha_{n}\right\}$ is a real sequence in $(0,1)$ satisfying suitable conditions. Then it is proved that $\left\{x_{n}\right\}$ converges strongly to the unique point $x^{*} \in A^{-1}(0)$. Finally, our theorems are applied to the convex minimization problem.
\end{abstract}

Keywords: Strongly monotone, Lipschitz, Bounded

\section{Background}

Let $H$ be a real Hilbert space with inner product $\langle\cdot, \cdot\rangle_{H}$ and norm $\|\cdot\|_{H}$. An operator $A: H \rightarrow H$ is called monotone if

$$
\langle A x-A y, x-y\rangle_{H} \geq 0 \forall x, y \in H,
$$

and is called strongly monotone if there exists $k \in(0,1)$ such that

$$
\langle A x-A y, x-y\rangle_{H} \geq k\|x-y\|_{H}^{2} \forall x, y \in H .
$$

Interest in monotone operators stems mainly from their usefulness in numerous applications. Consider, for example, the following: Let $f: H \rightarrow \mathbb{R} \cup\{\infty\}$ be a proper lower semi continuous and convex function. The subdifferential, $\partial f: H \rightarrow 2^{H}$ of $f$ at $x \in H$ is defined by

$$
\partial f(x)=\left\{x^{*} \in H: f(y)-f(x) \geq\left\langle y-x, x^{*}\right\rangle \forall y \in H\right\} .
$$

It is easy to check that $\partial f: H \rightarrow 2^{H}$ is a monotone operator on $H$, and that $0 \in \partial f(x)$ if and only if $x$ is a minimizer of $f$. Setting $\partial f \equiv A$, it follows that solving the inclusion $0 \in A u$, in this case, is solving for a minimizer of $f$.

(c) 2015 Diop et al. This article is distributed under the terms of the Creative Commons Attribution 4.0 International License (http:// creativecommons.org/licenses/by/4.0/), which permits unrestricted use, distribution, and reproduction in any medium, provided you give appropriate credit to the original author(s) and the source, provide a link to the Creative Commons license, and indicate if changes were made. 
Several existence theorems have been established for the equation $A u=0$ when $A$ is of the monotone-type (see e.g., Deimling 1985; Pascali and Sburian 1978).

The extension of the monotonicity definition to operators from a Banach space into its dual has been the starting point for the development of nonlinear functional analysis. The monotone maps constitute the most manageable class because of the very simple structure of the monotonicity condition. The monotone mappings appear in a rather wide variety of contexts since they can be found in many functional equations. Many of them appear also in calculus of variations as subdifferential of convex functions. (Pascali and Sburian 1978, p. 101).

Let $E$ be a real normed space, $E^{*}$ its topological dual space. The map $J: E \rightarrow 2^{E^{*}}$ defined by

$$
J x:=\left\{x^{*} \in E^{*}:\left\langle x, x^{*}\right\rangle=\|x\| \cdot\left\|x^{*}\right\|,\|x\|=\left\|x^{*}\right\|\right\}
$$

is called the normalized duality map on $E$. where, $\langle$,$\rangle denotes the generalized duality$ pairing between $E$ and $E^{*}$.

A map $A: E \rightarrow E^{*}$ is called monotone if for each $x, y \in E$, the following inequality holds:

$$
\langle A x-A y, x-y\rangle \geq 0 .
$$

$A$ is called strongly monotone if there exists $k \in(0,1)$ such that for each $x, y \in E$, the following inequality holds:

$$
\langle A x-A y, x-y\rangle \geq k\|x-y\|^{2} .
$$

A map $A: E \rightarrow E$ is called accretive if for each $x, y \in E$, there exists $j(x-y) \in J(x-y)$ such that

$$
\langle A x-A y, j(x-y)\rangle \geq 0 .
$$

$A$ is called strongly accretive if there exists $k \in(0,1)$ such that for each $x, y \in E$, there exists $j(x-y) \in J(x-y)$ such that

$$
\langle A x-A y, j(x-y)\rangle \geq k\|x-y\|^{2} .
$$

In a Hilbert space, the normalized duality map is the identity map. Hence, in Hilbert spaces, monotonicity and accretivity coincide. For accretive-type operator $A$, solutions of the equation $A u=0$, in many cases, represent equilibrium state of some dynamical system (see e.g., Chidume 2009, p. 116).

For approximating a solution of $A u=0$, assuming existence, where $A: E \rightarrow E$ is of accretive-type, Browder (1967) defined an operator $T: E \rightarrow E$ by $T:=I-A$, where $I$ is the identity map on $E$. He called such an operator pseudo-contractive. It is trivial to observe that zeros of $A$ correspond to fixed points of $T$. For Lipschitz strongly pseudocontractive maps, Chidume (1987) proved the following theorem.

Theorem C1 (Chidume 1987) Let $E=L_{p}, 2 \leq p<\infty$, and $K \subset E$ be nonempty closed convex and bounded. Let $T: K \rightarrow K$ be a strongly pseudo-contractive and Lipschitz map. For arbitrary $x_{0} \in K$, let a sequence $\left\{x_{n}\right\}$ be defined iteratively by $x_{n+1}=\left(1-\lambda_{n}\right) x_{n}+\lambda_{n} T x_{n}, n \geq 0$, where $\left\{\lambda_{n}\right\} \subset(0,1)$ satisfies the following conditions: 
(i) $\sum_{n=1}^{\infty} \lambda_{n}=\infty$, (ii) $\sum_{n=1}^{\infty} \lambda_{n}^{2}<\infty$. Then, $\left\{x_{n}\right\}$ converges strongly to the unique fixed point of $T$.

By setting $T:=I-A$ in Theorem $\mathrm{C} 1$, the following theorem for approximating a solution of $A u=0$ where $A$ is a strongly accretive and bounded operator can be proved.

Theorem C2 Let $E=L_{p}, 2 \leq p<\infty$. Let $A: E \rightarrow E$ be a strongly accretive and bounded map. Assume $A^{-1}(0) \neq \emptyset$. For arbitrary $x_{0} \in K$, let a sequence $\left\{x_{n}\right\}$ be defined iteratively by $x_{n+1}=x_{n}-\lambda_{n} A x_{n}, n \geq 0$, where $\left\{\lambda_{n}\right\} \subset(0,1)$ satisfies the following conditions: (i) $\sum_{n=1}^{\infty} \lambda_{n}=\infty$, (ii) $\sum_{n=1}^{\infty} \lambda_{n}^{2}<\infty$. Then, $\left\{x_{n}\right\}$ converges strongly to the unique solution of $A u=0$.

The main tool used in the proof of Theorem C1 is an inequality of Bynum (1976). This theorem signalled the return to extensive research efforts on inequalities in Banach spaces and their applications to iterative methods for solutions of nonlinear equations. Consequently, Theorem $\mathrm{C} 1$ has been generalized and extended in various directions, leading to flourishing areas of research, for the past thirty years or so, for numerous authors (see e.g., Censor and Reich 1996; Chidume 1986, 1987, 2002; Chidume and Bashir 2007; Chidume and Chidume 2005, 2006; Chidume and Osilike 1999; Deng 1993; Zhou and Jia 1996; Liou 1990; Qihou 1990; Reich 1977, 1978, 1979; Reich and Sabach 2009, 2010; Weng 1991; Xiao 1998; Xu 1991, 1991, 1992; Berinde and Maruster 2014; Moudafi 2003, 2004; 2010; Moudafi and Thera 1997; Xu and Roach 1991; Xu et al. 1995; Zhu 1994 and a host of other authors). Recent monographs emanating from these researches include those by Berinde (2007), Chidume (2009), Goebel and Reich (1984), and William and Shahzad (2014).

Unfortunately, the success achieved in using geometric properties developed from the mid 1980s to early 1990s in approximating zeros of accretive-type mappings has not carried over to approximating zeros of monotone-type operators in general Banach spaces. Part of the problem is that since $A$ maps $E$ to $E^{*}$, for $x_{n} \in E, A x_{n}$ is in $E^{*}$. Consequently, a recursion formula containing $x_{n}$ and $A x_{n}$ may not be well defined.

Attempts have been made to overcome this difficulty by introducing the inverse of the normalized duality mapping in the recursion formulas for approximating zeros of monotone-type mappings.

In this paper, we introduce an iterative scheme of Mann-type to approximate the unique zero of a strongly monotone bounded mapping in 2-uniformly convex real Banach with uniformly Gâteaux differentiable norm. Then we apply our results to the convex minimization problem. Finally, our method of proof is of independent interest.

Remark 1 In $L_{p}$ spaces, $1<p<\infty$, the formula for $J$ is known precisely (see e.g., Chidume 2009; Cioranescu 1990). In fact, from Cioranescu (1990), Corollary 4.10, p. 72, we have for $J: L_{p} \rightarrow L_{p}{ }^{*}, 1<p<\infty$,

$$
J(f)=|f|^{p-1} \cdot \operatorname{sign} \frac{f}{\|f\|^{p-1}} .
$$




\section{Preliminaries}

Let $E$ be a normed linear space. $E$ is said to be smooth if

$$
\lim _{t \rightarrow 0} \frac{\|x+t y\|-\|x\|}{t}
$$

exist for each $x, y \in S_{E}$ (Here $S_{E}:=\{x \in E:\|x\|=1\}$ is the unit sphere of $E$ ). $E$ is said to be uniformly smooth if it is smooth and the limit is attained uniformly for each $x, y \in S_{E}$, and $E$ is Fréchet differentiable if it is smooth and the limit is attained uniformly for $y \in S_{E}$.

A normed linear space $E$ is said to be strictly convex if:

$$
\|x\|=\|y\|=1, x \neq y \Rightarrow\left\|\frac{x+y}{2}\right\|<1 .
$$

The modulus of convexity of $E$ is the function $\delta_{E}:(0,2] \rightarrow[0,1]$ defined by:

$$
\delta_{E}(\epsilon):=\inf \left\{1-\frac{1}{2}\|x+y\|:\|x\|=\|y\|=1,\|x-y\| \geq \epsilon\right\} .
$$

$E$ is uniformly convex if and only if $\delta_{E}(\epsilon)>0$ for every $\epsilon \in(0,2]$. Let $p>1$. Then $E$ is said to be $p$-uniformly convex if there exists a constant $c>0$ such that $\delta_{E}(\epsilon) \geq c \epsilon^{p}$ for all $\epsilon \in(0,2]$. Observe that every $p$-uniformly convex space is uniformly convex.

It is well known that $E$ is smooth if and only if $J$ is single valued. Moreover, if $E$ is a reflexive smooth and strictly convex Banach space, then $J^{-1}$ is single valued, one-to-one, surjective and it is the duality mapping from $E^{*}$ into $E$. Finally, if $E$ has uniform Gâteaux differentiable norm, then $J$ is norm-to-weak $*$ uniformly continuous on bounded sets.

In the sequel, we shall need the following results and definitions.

Theorem 2.1 (Xu 1991) Let $p>1$ be a given real number. Then the following are equivalent in a Banach space:

(i) E is p-uniformly convex.

(ii) There is a constant $c_{1}>0$ such that for every $x, y \in E$ and $j_{x} \in J_{p}(x)$, The following inequality holds:

$$
\|x+y\|^{p} \geq\|x\|^{p}+p\left\langle y, j_{x}\right\rangle+c_{1}\|y\|^{p} .
$$

(iii) There is a constant $c_{2}>0$ such that for every $x, y \in E$ and $j_{x} \in J_{p}(x), j_{y} \in J_{p}(y)$, the following inequality holds:

$$
\left\langle x-y, j_{x}-j_{y}\right\rangle \geq c_{2}\|x-y\|^{p} .
$$

Corollary 2.2 Let E be a 2-uniformly convex and smooth real Banach space. Then $J^{-1}$ is Lipschtzian form $E^{*}$ into $E$, i.e., there exists constant $L>0$ such for all $u, v \in E^{*}$ the following holds

$$
\left\|J^{-1} u-J^{-1} v\right\| \leq L\|u-v\| .
$$

Proof This follows from inequality (iii) of Theorem 2.1 with $p=2$. 
Let $E$ be a smooth real Banach space with dual $E^{*}$. The function $\phi: E \times E \rightarrow \mathbb{R}$, defined by

$$
\phi(x, y)=\|x\|^{2}-2\langle x, J y\rangle+\|y\|^{2}, x, y \in E,
$$

where $J$ is the normalized duality mapping from $E$ into $E^{*}$, introduced by Alber has been studied by Alber (1996), Alber and Guerre-Delabiere (2001), Kamimura and Takahashi (2002), Reich (1979) and a host of other authors. This functional $\phi$ will play a central role in what follows. If $E=H$, a real Hilbert space, then Eq. (2.3) reduce to $\phi(x, y)=\|x-y\|^{2}$ for $x, y \in H$. It is obvious from the definition of the function $\phi$ that

$$
(\|x\|-\|y\|)^{2} \leq \phi(x, y) \leq(\|x\|+\|y\|)^{2} \forall x, y \in E .
$$

Define a functional $V: E \times E^{*} \rightarrow \mathbb{R}$ by

$$
V\left(x, x^{*}\right)=\|x\|^{2}-2\left\langle x, x^{*}\right\rangle+\left\|x^{*}\right\|^{2}, x \in E, x^{*} \in E^{*} .
$$

Then, it is easy to see that

$$
V\left(x, x^{*}\right)=\phi\left(x, J^{-1} x^{*}\right) \forall x \in E, x^{*} \in E^{*} .
$$

Lemma 2.3 (Alber 1996) Let $E$ be a reflexive strictly convex and smooth real Banach space with $E^{*}$ as its dual. Then,

$$
V\left(x, x^{*}\right)+2\left\langle J^{-1} x^{*}-x, y^{*}\right\rangle \leq V\left(x, x^{*}+y^{*}\right)
$$

for all $x \in E$ and $x^{*}, y^{*} \in E^{*}$.

Lemma 2.4 (Kamimura and Takahashi 2002) Let E be a smooth uniformly convex real Banach space, and let $\left\{x_{n}\right\}$ and $\left\{y_{n}\right\}$ be two sequences of $E$. If either $\left\{x_{n}\right\}$ or $\left\{y_{n}\right\}$ is bounded and $\phi\left(x_{n}, y_{n}\right) \rightarrow 0$ as $n \rightarrow \infty$, then $\left\|x_{n}-y_{n}\right\| \rightarrow 0$ as $n \rightarrow \infty$.

Lemma 2.5 (Tan and $\mathrm{Xu}$ 1993) Let $\left\{a_{n}\right\}$ be a sequence of non-negative real numbers satisfying the following relation:

$$
a_{n+1} \leq a_{n}+\sigma_{n} n \geq 0 .
$$

Such that $\sum_{n=0}^{\infty} \sigma_{n}<\infty$. Then $\lim _{n \rightarrow \infty} a_{n}$ exists. If addition, the sequence $\left\{a_{n}\right\}$ has a subsequence that converges to 0 . Then $\left\{a_{n}\right\}$ converges to 0 .

The following results will be useful.

Lemma 2.6 (Alber and Ryazantseva 2006) For $p>1$, let $X$ be a $p$-uniformly convex and smooth real Banach space and $S$ a bounded subset of $X$. Then there exists a positive constant $\alpha$ such that

$$
\alpha\|x-y\|^{p} \leq \phi(x, y) \forall x, y \in S .
$$

Lemma 2.7 Let E be a 2-uniformly convex smooth real Banach space. Then the following inequality holds:

$$
\|x-y\|^{2} \geq \phi(x, y)+\left(c_{1}-1\right)\|x\|^{2} x, y \in E,
$$


where $0 \leq c_{1} \leq 1$ has the same meaning as in Theorem 2.1 .

Proof Using (ii) of Theorem 2.1, we have

$$
\|x-y\|^{2} \geq\|x\|^{2}-2\langle y, J x\rangle+c_{1}\|y\|^{2} .
$$

Interchanging $x$ and $y$, we obtain

$$
\begin{aligned}
\|x-y\|^{2} & \geq\|y\|^{2}-2\langle x, J y\rangle+c_{1}\|x\|^{2} \\
& =\phi(x, y)+\left(c_{1}-1\right)\|x\|^{2} .
\end{aligned}
$$

\section{Main results}

We now prove the following result

Theorem 3.1 Let E be a 2-uniformly convex real Banach space with uniformly Gâteaux differentiable norm and $E^{*}$ its dual space. Let $A: E \rightarrow E^{*}$ be a bounded and k-strongly monotone mapping such that $A^{-1}(0) \neq \emptyset$. For arbitrary $x_{1} \in E$, let $\left\{x_{n}\right\}$ be the sequence defined iteratively by:

$$
x_{n+1}=J^{-1}\left(J x_{n}-\alpha_{n} A x_{n}\right), n \geq 1,
$$

where $J$ is the normalized duality mapping from $E$ into $E^{*}$ and $\left\{\alpha_{n}\right\} \subset(0,1)$ is a real sequence satisfying the following conditions: ( $i) \sum_{n=1}^{\infty} \alpha_{n}=\infty$; (ii) $\sum_{n=0}^{\infty} \alpha_{n}^{2}<\infty$. Then, there exists $\gamma_{0}>0$ such that if $\alpha_{n}<\gamma_{0}$, the sequence $\left\{x_{n}\right\}$ converges strongly to the unique solution of the equation $A u=0$.

Proof The proof is in two steps:

Step 1: We prove that $\left\{x_{n}\right\}$ is bounded. Since $A^{-1}(0) \neq \emptyset$, let $x^{*} \in A^{-1}(0)$.There exists $r>0$ such that:

$$
r \geq \max \left\{4\left(1-c_{1}\right)\left\|x^{*}\right\|^{2}, \phi\left(x_{1}, x^{*}\right)\right\} .
$$

We show that $\phi\left(x_{n}, x^{*}\right) \leq r$ for all $n \geq 1$. The proof is by induction. We have $\phi\left(x_{1}, x^{*}\right) \leq r$. Assume that $\phi\left(x_{n}, x^{*}\right) \leq r$ for some $n \geq 1$. We show that $\phi\left(x_{n+1}, x^{*}\right) \leq r$. From the induction assumption and Lemma 2.6, there exists $\alpha^{*}>0$ such that $\left\|x_{n}-x^{*}\right\|^{2} \leq r \alpha^{*}$. Since $A$ is bounded, we have:

$$
M_{0}=2 L \sup \left\{\|A x\|^{2},\left\|x-x^{*}\right\| \leq \sqrt{r \alpha^{*}}\right\}+1<\infty,
$$

where $L$ is a Lipschitz constant of $J^{-1}$. Define

$$
\gamma_{0}=\min \frac{1}{2}\left\{1, \frac{k r}{M_{0}}\right\}
$$

Using the definition of $x_{n+1}$, we compute as follows: 


$$
\begin{aligned}
\phi\left(x^{*}, x_{n+1}\right) & =\phi\left(x^{*}, J^{-1}\left(J x_{n}-\alpha_{n} A x_{n}\right)\right) \\
& =V\left(x^{*}, J x_{n}-\alpha_{n} A x_{n}\right) .
\end{aligned}
$$

Using Lemma 2.3, with $y^{*}=\alpha_{n} A x_{n}$, we have:

$$
\begin{aligned}
\phi\left(x^{*}, x_{n+1}\right)= & V\left(x^{*}, J x_{n}-\alpha_{n} A x_{n}\right) \\
\leq & V\left(x^{*}, J x_{n}\right)-2 \alpha_{n}\left\langle J^{-1}\left(J x_{n}-\alpha_{n} A x_{n}\right)-x^{*}, A x_{n}-A x^{*}\right\rangle \\
= & \phi\left(x^{*}, x_{n}\right)-2 \alpha_{n}\left\langle x_{n}-x^{*}, A x_{n}-A x^{*}\right\rangle-2 \alpha_{n}\left\langle J^{-1}\left(J x_{n}-\alpha_{n} A x_{n}\right)-x_{n}, A x_{n}\right\rangle \\
= & \phi\left(x^{*}, x_{n}\right)-2 \alpha_{n}\left\langle x_{n}-x^{*}, A x_{n}-A x^{*}\right\rangle \\
& -2 \alpha_{n}\left\langle J^{-1}\left(J x_{n}-\alpha_{n} A x_{n}\right)-J^{-1}\left(J x_{n}\right), A x_{n}\right\rangle .
\end{aligned}
$$

Using the strong monotonocity of $A$, Schwartz inequality and the Lipzchitz property of $J^{-1}$, we obtain

$$
\begin{aligned}
\phi\left(x^{*}, x_{n+1}\right) & \leq \phi\left(x^{*}, x_{n}\right)-2 \alpha_{n} k\left\|x_{n}-x^{*}\right\|^{2}+2 \alpha_{n}\left\|J^{-1}\left(J x_{n}-\alpha_{n} A x_{n}\right)-J^{-1}\left(J x_{n}\right)\right\|\left\|A x_{n}\right\| \\
& \leq \phi\left(x^{*}, x_{n}\right)-2 \alpha_{n} k\left\|x_{n}-x^{*}\right\|^{2}+2 \alpha_{n}^{2} L\left\|A x_{n}\right\|^{2} .
\end{aligned}
$$

Using Lemma 2.7, it follows that

$$
\phi\left(x^{*}, x_{n+1}\right) \leq \phi\left(x^{*}, x_{n}\right)-2 \alpha_{n} k \phi\left(x^{*}, x_{n}\right)+2 \alpha_{n} k\left(1-c_{1}\right)\left\|x^{*}\right\|^{2}+\alpha_{n}^{2} M_{0} .
$$

Finally, using inequality (3.2), the definition of $\gamma_{0}$ (3.4), and the induction assumption, we have

$$
\begin{aligned}
\phi\left(x^{*}, x_{n+1}\right) & \leq\left(1-2 k \alpha_{n}\right) r+\alpha_{n} k \frac{r}{2}+\alpha_{n} k \frac{r}{2} \\
& \leq\left(1-k \alpha_{n}\left(2-\frac{1}{2}-\frac{1}{2}\right)\right) r \\
& \leq\left(1-k \alpha_{n}\right) r .
\end{aligned}
$$

Therefore, $\phi\left(x^{*}, x_{n+1}\right) \leq r$. Thus, by induction, $\phi\left(x^{*}, x_{n}\right) \leq r$ for all $n \geq 1$. So, by inequality (2.4), $\left\{x_{n}\right\}$ is bounded.

Step 2: We now prove that $\left\{x_{n}\right\}$ converges strongly to the unique point $x^{*}$ of $A^{-1}(0)$. Following the same arguments as in Step 1, using the fact the sequence $\left\{x_{n}\right\}$ is bounded and $A$ is bounded, there exists a positive constant $M$ such that

$$
\phi\left(x^{*}, x_{n+1}\right) \leq \phi\left(x^{*}, x_{n}\right)-2 \alpha_{n} k\left\|x_{n}-x^{*}\right\|^{2}+\alpha_{n}^{2} M .
$$

Therefore,

$$
\phi\left(x^{*}, x_{n+1}\right) \leq \phi\left(x^{*}, x_{n}\right)+\alpha_{n}^{2} M .
$$

Using the hypothesis $\sum_{n=0}^{\infty} \alpha_{n}^{2}<\infty$ and Lemma 2.5, it follows that $\lim _{n \rightarrow \infty} \phi\left(x^{*}, x_{n}\right)$ exists.
From (3.6), we have

$$
\sum_{n=1}^{\infty} \alpha_{n}\left\|x_{n}-x^{*}\right\|<\infty
$$


Using the fact that $\sum_{n=0}^{\infty} \alpha_{n}=\infty$, it follows that lim inf $\left\|x^{*}-x_{n}\right\|^{2}=0$. Therefore, there exists a subsequence $\left\{x_{n_{k}}\right\}$ of $\left\{x_{n}\right\}$ such that $x_{n_{k}} \rightarrow x^{*}$ as $k \rightarrow \infty$. We have

$$
\phi\left(x^{*}, x_{n_{k}}\right)=\|x\|^{2}-2\left\langle x^{*}, J x_{n_{k}}\right\rangle+\left\|x_{n_{k}}\right\|^{2} .
$$

Since $\left\{x_{n}\right\}$ is bounded and $J$ is norm-to weak* uniformly continuous on bounded subsets of $E$, it follows that $\left\{\phi\left(x^{*}, x_{n}\right)\right\}$ has a subsequence that converges to 0 . Thus, by Lemma (), $\left\{\phi\left(x^{*}, x_{n}\right)\right\}$ converges strongly to 0 . Applying Lemma(), we obtain that $\left\|x_{n}-x^{*}\right\| \rightarrow 0$ as $n \rightarrow \infty$. This completes the proof.

Corollary 3.2 Let $E=L_{p}, 1<p \leq 2$ and $A: E \rightarrow E^{*}$ be a bounded and strongly monotone mapping. For arbitrary $x_{1} \in E$, let $\left\{x_{n}\right\}$ be the sequence defined iteratively by:

$$
x_{n+1}=J^{-1}\left(J x_{n}-\alpha_{n} A x_{n}\right), n \geq 1,
$$

where $J$ is the normalized duality mapping from $E$ into $E^{*}$ and $\left\{\alpha_{n}\right\} \subset(0,1)$ is a real sequence satisfying the following conditions: ( $i) \sum_{n=1}^{\infty} \alpha_{n}=\infty$; (ii) $\sum_{n=0}^{\infty} \alpha_{n}^{2}<\infty$.

Then, there exists $\gamma_{0}>0$ such that if $\alpha_{n}<\gamma_{0}, \forall n \geq 1$ the sequence $\left\{x_{n}\right\}$ converges strongly to the unique solution of the equation $A u=0$.

Proof Since $L_{p}$ spaces, $1<p \leq 2$ are 2-uniformly convex Banach space with uniformly Gâteaux differentiable norm, then the proof follows from Theorem 3.1.

\section{Application to convex minimization problems}

In this section, we study the problem of finding a minimizer of a convex function $f$ defined from a real Banach space $E$ to $\mathbb{R}$.

The following basic results are well known.

Lemma 4.1 Let $f: E \rightarrow \mathbb{R}$ be a real-valued differentiable convex function and $a \in E$. $d f: E \rightarrow E^{*}$ denotes the differential map associated to $f$. Then the following hold.

1. The point $a$ is a minimizer of $f$ on $E$ if and only if $d f(a)=0$.

2. If $f$ is bounded, then $f$ is locally Lipschitzian, i.e., for every $x_{0} \in E$ and $r>0$, there exists $\gamma>0$ such that $f$ is $\gamma$-Lipschitzian on $B\left(x_{0}, r\right)$, i.e.

$$
|f(x)-f(y)| \leq \gamma\|x-y\| \forall x, y \in B\left(x_{0}, r\right) .
$$

Lemma 4.2 Let $E$ be normed linear space and $f: E \rightarrow \mathbb{R}$ a real-valued differentiable convex function. Assume that $f$ is bounded. Then the differential map $d f: E \rightarrow E^{*}$ is bounded.

Proof Let $x_{0} \in E$ and $r>0$. Set $B:=B\left(x_{0}, r\right)$. We show that $d f(B)$ is bounded. From lemma 4.1, there exists $\gamma>0$ such that

$$
|f(x)-f(y)| \leq \gamma\|x-y\| \forall x, y \in B .
$$


Let $z^{*} \in d f(B)$ and $x^{*} \in B$ such that $z^{*}=d f\left(x^{*}\right)$. Since $B$ is open, for all $u \in E$, there exists $t>0$ such that $x^{*}+t u \in B$. Using the fact that $z^{*}=d f\left(x^{*}\right)$ the convexity of $f$ and inequality (4.1), it follows that

$$
\begin{aligned}
\left\langle z^{*}, t u\right\rangle & \leq f\left(x^{*}+t u\right)-f\left(x^{*}\right) \\
& \leq t \gamma\|u\|
\end{aligned}
$$

so that

$$
\left\langle z^{*}, u\right\rangle \leq \gamma\|u\| \forall u \in E .
$$

Therefore $\left\|z^{*}\right\| \leq \gamma$. Hence $d f(B)$ is bounded.

Definition 4.3 A function $f: E \rightarrow \mathbb{R}$ is said to be strongly convex if there exists $\alpha>0$ such that for every $x, y \in E$ with $x \neq y$ and $\lambda \in(0,1)$, the following inequality holds:

$$
f(\lambda x+(1-\lambda) y) \leq \lambda f(x)+(1-\lambda) f(y)-\alpha\|x-y\|^{2} .
$$

Lemma 4.4 Let $E$ be normed linear space and $f: E \rightarrow \mathbb{R}$ a real-valued differentiable convex function. Assume that $f$ is strongly convex. Then the differential map $d f: E \rightarrow E^{*}$ is strongly monotone, i.e., there exists a positive constant $k$ such that

$$
\langle d f(x)-d f(y), x-y\rangle \geq k\|x-y\|^{2} \forall x, y \in E .
$$

We now prove the following theorem.

Theorem 4.5 Let E be a 2-uniformly convex real Banach space with uniformly Gâteaux differentiable norm and let $f: E \rightarrow \mathbb{R}$ be a differentiable, bounded, strongly convex realvalued function which satisfies the growth condition: $f(x) \rightarrow+\infty$ as $\|x\| \rightarrow+\infty$. For arbitrary $x_{1} \in E$, let $\left\{x_{n}\right\}$ be the sequence defined iteratively by:

$$
x_{n+1}=J^{-1}\left(J x_{n}-\alpha_{n} d f\left(x_{n}\right)\right), n \geq 1,
$$

where $J$ is the normalized duality mapping from $E$ into $E^{*}$ and $\left\{\alpha_{n}\right\} \subset(0,1)$ is a real sequence satisfying the following conditions: ( $i) \sum_{n=1}^{\infty} \alpha_{n}=\infty$; (ii) $\sum_{n=0}^{\infty} \alpha_{n}^{2}<\infty$. Then, fhas a unique minimizer $a^{*} \in E$ and there exists $\gamma_{0}>0$ such that if $\alpha_{n}<\gamma_{0}$, the sequence $\left\{x_{n}\right\}$ converges strongly to $a^{*}$.

Proof Since $E$ is reflexive, then from the growth condition, the continuity and the strict convexity of $f, f$ has a unique minimizer $a^{*}$ characterized by $d f\left(a^{*}\right)=0$ (Lemma 4.1). Finally, from Lemmas 4.2 and 4.4, the differential map $d f: E \rightarrow E^{*}$ is bounded and strongly monotone. Therefore, the proof follows from Theorem 3.1.

\section{Conclusion}

In this work, we proposed a new iteration scheme for the approximation of zeros of monotone mappings defined in certain Banach spaces. Our results are used to approximate minimizers of convex functions. The results obtained in this paper are important improvements of recent important results in this field. 
Authors' contributions

The authors, CD, TMMS, ND and CEC with the consultation of each other carried out this work. All authors read and approved the final manuscript.

\section{Author details}

${ }^{1}$ Gaston Berger University, Saint Louis, Senegal. ${ }^{2}$ Department of Mathematics, African University of Sciences and Technology, Abuja, Nigeria.

\section{Compliance with ethical guidelines}

Competing interests

The authors declare that they have no competing interests.

Received: 8 June 2015 Accepted: 20 July 2015

Published online: 28 July 2015

\section{References}

Alber Y (1996) Metric and generalized projection operator in banach space: properties and applications. In: Kartsatos AG (ed) Theory and applications of nonlinear operators of accretive and monotone type. Marcel Dekker, New York, pp 15-50

Alber Y, Guerre-Delabiere S (2001) On the projection methods for fixed point problems. Analysis (Munich) 21(1):17-39

Alber Y, Ryazantseva I (2006) Nonlinear ILL-posed problems of monotone type. Springer, Dordrecht

Berinde $V$ (2007) Iterative approximation of fixed points, lecture notes in mathematics. Springer, London

Berinde V, Maruster S, Rus IA (2014) An abstract point of view on iterative approximation of fixed points of nonself operators. J Nonlinear Convex Anal 15(5):851-865

Browder FB (1967) Nonlinear mappings of nonexpansive and accretive type in Banach spaces. Bull Am Math Soc 73:875-882

Bynum WL (1976) Weak parallelogram laws for Banach spaces. Can Math Bull 19(3):269-275

Censor Y, Reich R (1996) Iterations of paracontractions and firmly nonexpansive operators with applications to feasibility and optimization. Optimization 37(4):323-339

Cioranescu I (1990) Geometry of Banach spaces, duality mappings and nonlinear problems, vol 62. Kluwer Academic Publishers, Dordrecht

Chidume CE (1986) An approximation method for monotone Lipschitzian operators in Hilbert-spaces. JJ Aust Math Soc Ser A Pure Math Stat 41:59-63

Chidume CE (2002) Convergence theorems for asymptotically pseudo-contractive mappings. Nonlinear Anal Theory Methods Appl 49(1):1-11

Chidume CE (2009) Properties Geometric, of Banach Spaces and Nonlinear iterations, vol 1965, of lectures notes in mathematics. Springer, London

Chidume CE (1987) Iterative approximation of fixed points of Lipschitzian strictly pseudo-contractive mappings. Proc Am Math Soc 99(2):283-288

Chidume CE, Bashir A (2007) Approximation of common fixed points for finite families of nonself asymptotically nonexpansive mappings in Banach spaces. J Math Anal Appl 326:960-973

Chidume CE, Chidume CO (2005) Convergence theorems for fixed points of uniformly continuous generalized Phi-hemicontractive mappings. J Math Anal Appl 303:545-554

Chidume CE, Chidume CO (2006) Convergence theorem for zeros of generalized Phi-quasi-accretive operators. Proc Am Math Soc 134:243-251

Chidume CE, Osilike MO (1999) Iterative solutions of nonlinear accretive operator equations in arbitrary Banach spaces. Nonlinear Anal Theory Methods Appl 36:863-872

Deimling K (1985) Nonlinear functional analysis. Springer, New York

Deng L (1993) On Chidume's open question. J Math Appl 174(2):441-449

Goebel K, Reich S (1984) Uniform convexity, hyperbolic geometry, and nonexpansive mappings. Monographs and textbooks in pure and applied mathematics, vol 83. Marcel Dekker, Inc., New York

Kamimura S, Takahashi W (2002) Strong convergence of proximal-type algorithm in Banach space. SIAMJ Optim 13(3):938-945

Liou L (1990) Ishikawa and Mann iterative for hemi-contractive mapping. J Math Anal Appl 48:55-62

Moudafi A (2003) A relaxed alternating CQ-algorithm for convex feasibility problems. Nonlinear Anal 79:117-121

Moudafi A (2004) Alternating CQ-algorithm for convex feasibility and split fixed-point problems. J Nonlinear Convex Anal 15(4):809-818

Moudafi A (2010) Proximal methods for a class of bilevel monotone equilibrium problems. J Glob Optim 47(2):45-52

Moudafi A, Thera M (1997) Finding a zero of the sum of two maximal monotone operators. J Optim Theory Appl 94(2):425-448

Pascali D, Sburian S (1978) Nonlinear mappings of monotone type. Editura Academia Bucuresti, Romania

Qihou $L$ (1990) The convergence theorems of the sequence of Ishikawa iterates for hemi-contractive mapping. J Math Anal Appl 148:55-62

Reich S (1979) Constructive techniques for accretive and monotone operators, applied non-linear analysis. Academic Press, New York, pp 335-345

Reich S (1977) Extension problems for accretive sets in Banach spaces. J Funct Anal 26:378-395

Reich S (1978) Iterative methods for accretive sets in Banach Spaces. Academic Press, New York, pp 317-326 
Reich S, Sabach S (2009) A strong convergence theorem for a proximal-type algorithm in reflexive Banach spaces. J Nonlinear Convex Anal 10(3):471-485

Reich S, Sabach S (2010) Two strong convergence theorems for a proximal method in reflexive Banach spaces. Numer Funt Anal Optim 31(1--3):22-44

Tan HK, Xu HK (1993) Approximating fixed points of nonexpansive mappings by the Ishikawa iteration process. J Math Anal Appl 178:301-308

Weng XL (1991) Fixed point iteration for local striclty pseudo-contractive mappings. Proc Am Math Soc 113(3):727-731

William K, Shahzad N (2014) Fixed point theory in distance spaces. Springer, New York

Xiao R (1998) Chidume's open problems and fixed point theorems. Xichuan Daxue Xuebao 35(4):505-508

Xu HK (1991) Inequalities in Banach spaces with applications. Nonlinear Anal 16(12):1127-1138

Xu Y (1991) Existence and convergence for fixed points of mappings of the asymptotically nonexpansive type. Nonlinear Anal 16:1139-1146

Xu ZB (1992) A note on the Ishikawa iteration schemes. J Math Anal Appl 167:582-587

Xu ZB, Jiang YL, Roach GF (1995) A further necessary and sufficient condition for strong convergence of nonlinear contraction semigroups and of iteration methods for accretive operators in Banach spaces. Proc Edinburgh Math Soc 38(2):1-12

Xu Z, Roach GF (1991) Characteristic inequalities for uniformly convex and uniformly smooth Banach space. J Math Anal Appl 157:189-210

Zhou H, Jia Y (1996) Approximating the zeros of accretive operators by the Ishikawa iteration process. Abstr Appl Anal 1(2):153-167

Zhu L (1994) Iteration solution of nonlinear equations involving m-accretive operators in Banach spaces. J Math Anal Appl 188:410-415

\section{Submit your manuscript to a SpringerOpen ${ }^{\circ}$ journal and benefit from:}

- Convenient online submission

- Rigorous peer review

- Immediate publication on acceptance

- Open access: articles freely available online

- High visibility within the field

- Retaining the copyright to your article

Submit your next manuscript at $\mathbf{s p r i n g e r o p e n . c o m ~}$ 